\title{
Weighted Finite Automata over Strong Bimonoids
}

\author{
Manfred Droste $^{\mathrm{a}} \quad$ Torsten Stüber $^{\mathrm{b}, *} \quad$ Heiko Vogler ${ }^{\mathrm{b}}$ \\ a Institut für Informatik, Universität Leipzig \\ D-04009 Leipzig, Germany \\ droste@informatik.uni-leipzig.de \\ ${ }^{\mathrm{b}}$ Faculty of Computer Science, Technische Universität Dresden \\ D-01062 Dresden, Germany \\ \{stueber, vogler\}@tcs.inf.tu-dresden.de
}

September 9, 2008

\begin{abstract}
We investigate weighted finite automata over strings and strong bimonoids. Such algebraic structures satisfy the same laws as semirings except that no distributivity laws need to hold. We define two different behaviors and prove precise characterizations for them if the underlying strong bimonoid satisfies local finiteness conditions. Moreover, we show that in this case the given weighted automata can be determinized.
\end{abstract}

\section{Introduction}

In the seminal paper [30], Schützenberger extended Kleene's classical result on the coincidence between recognizable and rational languages to the realm of weighted automata, their behaviors, and rational formal power series. Weighted finite automata are classical nondeterministic automata in which the transitions carry weights. These weights may model, e.g., the amount of recources needed for the execution of a transition, or the probability of its successful execution. The weights can be taken from any semiring, therefore weighted automata have both a rich structure theory $[5,10,20,28,29,33]$ as well as practical applications in digital image compression $[1,8,11,14,16]$, natural language processing $[7,18$, 25,27 , and probabilistic model checking [2]. In semirings, by definition, the multiplication operation is distributive over addition and this was crucial for almost all of the theory developed so far.

It is the goal of this paper to investigate automata with weights in strong bimonoids; these can be viewed as semirings where the distributivity assumption is dropped. Trivially, all semirings are bimonoids, but there are also many

\footnotetext{
${ }^{*}$ The work of this author was partially supported by Deutsche Forschungsgemeinschaft, project DFG VO 1011/4-1.
} 
natural examples of bimonoids which are not semirings like, e.g., the tropical bimonoid and near-semirings (see Example 1), the interval $[0,1]$ with t-conorm and t-norm from multi-valued logic [17], the "string semiring" of all words over an alphabet arising in natural language processing [26], or the algebraic cost structure from algebraic path problems [21].

The main results of this paper are as follows. First, we define for every weighted finite automaton $M$ over some bimonoid $A$ two different kinds of behaviors, called the run semantics and the initial algebra semantics. Both of them are functions assigning to each word $w$ an element of $A$ as value, the weight obtained when executing $M$ on $w$. We show that these two semantics coincides if and only if $A$ is right-distributive (cf. Lemma 6).

Secondly, if the addition and multiplication operation of $A$ are each locally finite (meaning that finitely generated submonoids are finite), every weighted finite automaton over $A$ assumes only finitely many weights as values; moreover, each value is assumed on a recognizable language of words (cf. Theorem 13).

A fundamental result in classical automata theory states that each nondeterministic finite automaton can be transformed into an equivalent deterministic one. Here we investigate weighted versions of this result. We show that, for each weighted finite automaton, its run semantics can be recognized by a crispdeterministic weighted automaton if and only if $A$ is additively and multiplicatively locally finite (cf. Theorem 16). A corresponding result holds also with respect to the initial algebra semantics provided that $A$ is right-distributive (cf. Theorem 23).

These results generalize several theorems from the literature $[3,15,23,24]$ derived for automata over lattice-ordered monoids or semiring-reducts of residuated lattices which are particular semirings. They also apply, for instance, to all bounded lattices, even without distributivity assumption, since these lattices are additively and multiplicatively locally finite.

\section{Algebraic notions}

\subsection{Strong bimonoids and semirings}

A bimonoid is a structure $(A,+, \cdot, 0,1)$ consisting of a set $A$, two binary operations + and $\cdot$ on $A$ and two constants $0,1 \in A$ such that $(A,+, 0)$ and $(A, \cdot, 1)$ are monoids. As usual, we identify the structure $(A,+, \cdot, 0,1)$ with its carrier set $A$. We call $A$ a strong bimonoid if the operation + is commutative and 0 acts as multiplicative zero, i.e., $a \cdot 0=0=0 \cdot a$ for every $a \in A$. We say that a strong bimonoid $A$ is right distributive, if it satisfies $(a+b) \cdot c=a \cdot c+b \cdot c$ for every $a, b, c \in A$; we call $A$ left distributive, if $a \cdot(b+c)=a \cdot b+a \cdot c$ for every $a, b, c \in A$. Then a semiring is a strong bimonoid which is left and right distributive.

\section{Example 1.}

1. The tropical bimonoid is the strong bimonoid $\left(\mathbb{N}_{\infty},+, \min , 0, \infty\right)$ with $\mathbb{N}_{\infty}=\mathbb{N} \cup\{\infty\}$ and the usual extensions of + and min from $\mathbb{N}$ to $\mathbb{N}_{\infty}$. We note that it is not a semiring, because there are $a, b, c \in \mathbb{N}_{\infty}$ with $\min \{a, b+c\} \neq \min \{a, b\}+\min \{a, c\}$ (e.g., take $a=b=c \neq 0$ ).

2. The tropical semiring is the semiring $\left(\mathbb{N}_{\infty}, \min ,+, \infty, 0\right)$. 
3. The algebra $([0,1], \vee, \cdot, 0,1)$ with the usual multiplication $\cdot$ of real numbers is a strong bimonoid for, e.g., each of the following two definitions of $\vee$ for every $a, b \in[0,1]$ :

- $a \vee b=a+b-a \cdot b$ (called algebraic sum in [17]) and

- $a \vee b=\min \{a+b, 1\}$ (called bounded sum in [17]).

In neither of the two cases $([0,1], \vee, \cdot, 0,1)$ is a semiring.

4. Let $(C,+, 0)$ be a commutative monoid and let $A$ be the set of all mappings from $C$ into itself with pointwise addition, composition of mappings, constant mapping zero, and the identity mapping. Then $A$ constitutes a strong bimonoid satisfying only one distributivity law (which depends on the order used for defining the composition). Such structures are also called near semirings $[19,32]$.

5. Let $\Sigma$ be an alphabet. Consider the strong bimonoid $\left(\Sigma^{*} \cup\{\infty\}, \wedge, \cdot, \infty, \varepsilon\right)$ where $\wedge$ is the longest common prefix operation, $\cdot$ is the usual concatenation of words, and $\infty$ is a new element such that $w \wedge \infty=\infty \wedge w=w$ and $w \cdot \infty=\infty \cdot w=\infty$ for every $w \in \Sigma^{*} \cup\{\infty\}$. This bimonoid occurs in investigations for natural language processing, see [26]. It is clear that $\left(\Sigma^{*} \cup\{\infty\}, \wedge, \cdot, \infty, \varepsilon\right)$ is left distributive but not right distributive.

6. The Boolean semiring is the semiring $(\mathbb{B}, \vee, \wedge, 0,1)$ with $\mathbb{B}$ consisting of the truth values 0 and 1 , and $\vee$ and $\wedge$ are disjunction and conjunction, respectively.

7. We note that there are only two strong bimonoids with exactly two elements: the field with two elements and the Boolean semiring (since addition is determined by whether $1+1=0$ or $1+1=1$ ). However, there are strong bimonoids with 3 elements which are not semirings, take, e.g., $(\{0,1,2\}, \max , \hat{\bullet}, 0,1)$ where $a \hat{\imath} b=(a \cdot b)$ mod 3 for every $a, b \in\{0,1,2\}$.

8. Bounded lattices (lattices containing a greatest element 1 and a smallest element 0) are strong bimonoids. As is well known, there are large classes of lattices that are not distributive [13].

9. Moreover, bounded distributive lattices, semiring-reducts of semi-lattice ordered monoids and of complete residuated lattices, and Brouwerian lattices are semirings.

From now on and in the rest of the paper, we assume that $\Sigma$ is an arbitrary alphabet, i.e., a finite non-empty set, and $(A,+, \cdot, 0,1)$ denotes an arbitrary strong bimonoid unless specified otherwise.

\subsection{Formal power series}

A formal power series, for short, series, (over $\Sigma$ and $A$ ) is a mapping $\varphi: \Sigma^{*} \rightarrow$ $A$. Instead of $\varphi(w)$ we write $(\varphi, w)$ for every $w \in \Sigma^{*}$. The set of all series over $\Sigma$ and $A$ is denoted by $A\left\langle\left\langle\Sigma^{*}\right\rangle\right\rangle$. The image of $\varphi$ is the $\operatorname{set} \operatorname{im}(\varphi)=\{(\varphi, w) \in A \mid$ $\left.w \in \Sigma^{*}\right\}$. For every $a \in A$, we define $\varphi=a=\varphi^{-1}(a)=\left\{w \in \Sigma^{*} \mid(\varphi, w)=a\right\}$.

Let $a \in A$ and $\varphi \in A\left\langle\left\langle\Sigma^{*}\right\rangle\right\rangle$. The scalar multiplication of $a$ and $\varphi$ is the series $a \cdot \varphi \in A\left\langle\left\langle\Sigma^{*}\right\rangle\right\rangle$ defined by $(a \cdot \varphi, w)=a \cdot(\varphi, w)$ for every $w \in \Sigma^{*}$. 
Let $\varphi_{1}, \varphi_{2} \in A\left\langle\left\langle\Sigma^{*}\right\rangle\right\rangle$. The sum of $\varphi_{1}$ and $\varphi_{2}$ is the series $\varphi_{1}+\varphi_{2} \in A\left\langle\left\langle\Sigma^{*}\right\rangle\right\rangle$ defined by $\left(\varphi_{1}+\varphi_{2}, w\right)=\left(\varphi_{1}, w\right)+\left(\varphi_{2}, w\right)$ for every $w \in \Sigma^{*}$. The commutativity and associativity of the addition of $A$ carry over to the sum of series.

Let $L \subseteq \Sigma^{*}$. The characteristic function $\chi_{L} \in A\left\langle\left\langle\Sigma^{*}\right\rangle\right\rangle$ of $L$ is for every $w \in \Sigma^{*}$ defined as $\left(\chi_{L}, w\right)=1$ if $w \in L$ and $\left(\chi_{L}, w\right)=0$ otherwise.

\subsection{Matrices}

Let $P$ and $Q$ be sets. If $f: P \rightarrow Q$ and $g: Q \rightarrow R$ are functions, we denote their composition by $f ; g$ (i.e., apply first $f$, then $g$ ). We let $Q^{P}$ denote the set of all functions from $P$ to $Q$.

Let $Q$ be a finite non-empty set. A mapping $M: Q \times Q \rightarrow A$ is called a $Q \times Q$-matrix over $A$, and a mapping $v: Q \rightarrow A$ is called a $Q$-vector over $A$. For every $M \in A^{Q \times Q}, v \in A^{Q}$, and $q_{1}, q_{2} \in Q$ we write $M_{q_{1}, q_{2}}$ instead of $M\left(q_{1}, q_{2}\right)$, and $v_{q_{1}}$ instead of $v\left(q_{1}\right)$. If $A$ is a particular ordered set (e.g., the interval [0,1]), then matrices are called fuzzy relations, and vectors are called fuzzy subsets in the literature.

Now let $M_{1}, M_{2} \in A^{Q \times Q}$ and $v_{1}, v_{2} \in A^{Q}$. Then we define the matrix product $M_{1} \cdot M_{2} \in A^{Q \times Q}$, the matrix-vector products $v_{1} \cdot M_{1} \in A^{Q}$ and $M_{1} \cdot v_{1} \in A^{Q}$, and the scalar product $v_{1} \cdot v_{2} \in A$ as follows for every $q_{1}, q_{2} \in Q$ :

$$
\begin{aligned}
\left(M_{1} \cdot M_{2}\right)_{q_{1}, q_{2}} & =\sum_{q \in Q}\left(M_{1}\right)_{q_{1}, q} \cdot\left(M_{2}\right)_{q, q_{2}}, \\
\left(v_{1} \cdot M_{1}\right)_{q_{1}} & =\sum_{q \in Q}\left(v_{1}\right)_{q} \cdot\left(M_{1}\right)_{q, q_{1}} \\
\left(M_{1} \cdot v_{1}\right)_{q_{1}} & =\sum_{q \in Q}\left(M_{1}\right)_{q_{1}, q} \cdot\left(v_{1}\right)_{q} \\
v_{1} \cdot v_{2} & =\sum_{q \in Q}\left(v_{1}\right)_{q} \cdot\left(v_{2}\right)_{q} .
\end{aligned}
$$

Recall that the addition of $A$ is commutative and that $Q$ is non-empty; thus, the sums on the right-hand sides are well defined. We define the $Q$-unit matrix $I_{Q} \in A^{Q \times Q}$ as follows for every $q_{1}, q_{2} \in Q$ :

$$
\left(I_{Q}\right)_{q_{1}, q_{2}}= \begin{cases}1, & \text { if } q_{1}=q_{2} \\ 0, & \text { otherwise. }\end{cases}
$$

The following result is of fundamental importance in the theory of weighted automata over semirings; it is straightforward by elementary calculations.

Lemma 2. If $A$ is a semiring, then the matrix product and matrix-vector products (whenever defined) are associative, and $\left(A^{Q \times Q}, \cdot, I_{Q}\right)$ is a monoid.

Note that Lemma 2 fails in general, if $A$ is not left or not right distributive.

\section{$3 \quad$ Weighted finite automata}

In this section, we introduce weighted finite automata over bimonoids and different definitions of their behaviors (semantics). Then we investigate conditions under which these notions of behaviors coincide. Recall that $(A,+, \cdot, 0,1)$ is an arbitrary strong bimonoid. 
Definition 3. A pointed $\Sigma$-algebra is a triple $(P, \theta, q)$ such that $P$ is a set, $\theta: \Sigma \rightarrow P^{P}$ is a mapping, and $q \in P$. We define a mapping $h_{\theta}: \Sigma^{*} \rightarrow P$, called the successive evaluation of $\theta$, by letting $h_{\theta}(\varepsilon)=q$ and $h_{\theta}(w \sigma)=\theta(\sigma)\left(h_{\theta}(w)\right)$ for every $w \in \Sigma^{*}$ and $\sigma \in \Sigma$. We call $(P, \theta, q)$ finite if $P$ is finite.

In other words, if $w=\sigma_{1} \cdots \sigma_{n}$, then $h_{\theta}(w)=\left(\theta\left(\sigma_{1}\right) ; \ldots ; \theta\left(\sigma_{n}\right)\right)(q)$.

Definition 4. A weighted finite automaton (for short: wfa) over $\Sigma$ and $A$ is a quadruple $M=(Q, I, \tau, F)$ such that $Q$ is a finite non-empty set (of states), $I \in A^{Q}$ (initial weight vector), $\tau: \Sigma \rightarrow A^{Q \times Q}$ (transition mapping), and $F \in A^{Q}$ (final weight vector).

We define three different semantics for a wfa $M$.

Initial algebra semantics: We define the pointed $\Sigma$-algebra $\left(A^{Q}, \theta_{\tau}, I\right)$ by letting $\theta_{\tau}(\sigma)(v)=v \cdot \tau(\sigma)$ for every $\sigma \in \Sigma$ and $v \in A^{Q}$. The $i$-behavior of $M$, denoted by $\llbracket M \rrbracket_{i}$, is the series in $A\left\langle\left\langle\Sigma^{*}\right\rangle\right\rangle$ defined as follows for every $w \in$ $\Sigma^{*}:$

$$
\left(\llbracket M \rrbracket_{i}, w\right)=h_{\theta_{\tau}}(w) \cdot F .
$$

So, if $w=\sigma_{1} \cdots \sigma_{n}$, then $h_{\theta_{\tau}}(w)=\left(\ldots\left(\left(I \cdot \tau\left(\sigma_{1}\right)\right) \cdot \tau\left(\sigma_{2}\right)\right) \cdot \ldots \cdot \tau\left(\sigma_{n-1}\right)\right) \cdot$ $\tau\left(\sigma_{n}\right)$.

Run semantics: The $r$-behavior of $M$, denoted by $\llbracket M \rrbracket_{r}$, is the series in $A\left\langle\left\langle\Sigma^{*}\right\rangle\right\rangle$ defined for every $w=\sigma_{1} \cdots \sigma_{n} \in \Sigma^{*}$ by letting

$$
\left(\llbracket M \rrbracket_{r}, w\right)=\sum_{P \in Q^{n+1}} \operatorname{weight}_{M}(P, w),
$$

where for every $P=\left(q_{0}, \ldots, q_{n}\right) \in Q^{n+1}$ the weight weight $_{M}(P, w)$ of $P$ in $M$ for $w$ is defined as $I_{q_{0}} \cdot \tau\left(\sigma_{1}\right)_{q_{0}, q_{1}} \cdot \ldots \cdot \tau\left(\sigma_{n}\right)_{q_{n-1}, q_{n}} \cdot F_{q_{n}}$.

Free monoid semantics: Let $A$ be a semiring. Moreover, let $h_{\Sigma}$ be the unique monoid-morphism from the free monoid $\left(\Sigma^{*}, \cdot, \varepsilon\right)$ to $\left(A^{Q \times Q}, \cdot, I_{Q}\right)$ extending $\tau$ (cf. Lemma 2). Then the f-behavior of $M$, denoted by $\llbracket M \rrbracket_{f}$, is the series in $A\left\langle\left\langle\Sigma^{*}\right\rangle\right\rangle$ defined as follows for every $w \in \Sigma^{*}$ :

$$
\left(\llbracket M \rrbracket_{f}, w\right)=I \cdot h_{\Sigma}(w) \cdot F .
$$

Let $x \in\{i, r, f\}$. A series $\varphi \in A\left\langle\left\langle\Sigma^{*}\right\rangle\right\rangle$ is $x$-recognizable if there is a wfa $M$ over $\Sigma$ and $A$ such that $\llbracket M \rrbracket_{x}=\varphi$. We say that two wfa $M$ and $M^{\prime}$ over $\Sigma$ and $A$ are $x$-equivalent, if $\llbracket M \rrbracket_{x}=\llbracket M^{\prime} \rrbracket_{x}$.

We note that we will use the free monoid semantics only in Lemma 7 and Corollary 22 .

Example 5. Let $\Sigma=\{\sigma\}$. We consider the wfa $M=(Q, I, \tau, F)$ over $\Sigma$ and the tropical bimonoid $\left(\mathbb{N}_{\infty},+, \min , 0, \infty\right)$ with $Q=\{q, p\}, \tau(\sigma)_{p, p}=\tau(\sigma)_{p, q}=$ $\tau(\sigma)_{q, p}=\infty$ and $\tau(\sigma)_{q, q}=0$. Moreover, we define $I_{p}=1, F_{p}=\infty$ and $I_{q}=F_{q}=0$. If we neglect those transitions that have weight 0 , then we can illustrate $M$ as in Figure 1. Then, $\left(\llbracket M \rrbracket_{i}, \sigma^{n}\right)=\left(\llbracket M \rrbracket_{r}, \sigma^{n}\right)$ is the $n$th Fibonaccinumber, for every $n \geq 0$. We note that in [10] a similar automaton over the semiring of natural numbers has been used to define the Fibonacci-numbers. 


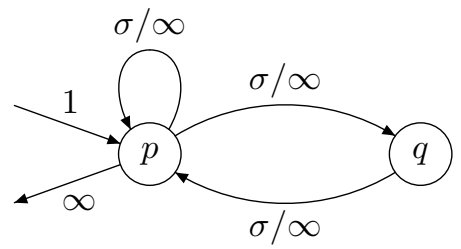

Figure 1: A wfa over the tropical bimonoid.

We will see later that, in general, the initial algebra semantics and the run semantics differ (cf. Examples 27 and 28).

Next we obtain a simple characterization when for every wfa $M$, its initial algebra semantics and its run semantics coincide.

Lemma 6. The following two statements are equivalent:

1. $A$ is right distributive.

2. $\llbracket M \rrbracket_{i}=\llbracket M \rrbracket_{r}$ for every wfa $M$ over $\Sigma$ and $A$.

Proof. 1. $\Rightarrow 2 .:$ Let $A$ be right distributive and let $M=(Q, I, \tau, F)$ and $w=\sigma_{1} \cdots \sigma_{n} \in \Sigma^{*}$. We show that $\left(\llbracket M \rrbracket_{i}, w\right)=\left(\llbracket M \rrbracket_{r}, w\right)$. To this end we show by induction on the length $n$ of $w$ that for every $q_{n} \in Q$ we have

$$
h_{\theta_{\tau}}(w)_{q_{n}}=\sum_{\left(q_{0}, \ldots, q_{n-1}\right) \in Q^{n}} I_{q_{0}} \cdot \tau\left(\sigma_{1}\right)_{q_{0}, q_{1}} \cdot \ldots \cdot \tau\left(\sigma_{n}\right)_{q_{n-1}, q_{n}} .
$$

Indeed, if $n=0$, then $w=\varepsilon$ and $h_{\theta_{\tau}}(\varepsilon)_{q_{0}}=I_{q_{0}}=\sum_{() \in Q^{0}} I_{q_{0}}$. Now let $n>0$ and $w=w^{\prime} \sigma_{n}$ for some $w^{\prime} \in \Sigma^{*}$ with $\left|w^{\prime}\right|=n-1$. Then

$$
\begin{aligned}
& h_{\theta_{\tau}}\left(w^{\prime} \sigma_{n}\right)_{q_{n}}=\theta_{\tau}\left(\sigma_{n}\right)\left(h_{\theta_{\tau}}\left(w^{\prime}\right)\right)_{q_{n}} \\
& =\left(h_{\theta_{\tau}}\left(w^{\prime}\right) \cdot \tau\left(\sigma_{n}\right)\right)_{q_{n}}=\sum_{q_{n-1} \in Q} h_{\theta_{\tau}}\left(w^{\prime}\right)_{q_{n-1}} \cdot \tau\left(\sigma_{n}\right)_{q_{n-1}, q_{n}} \\
& =\sum_{q_{n-1} \in Q} \sum_{\left(q_{0}, \ldots, q_{n-2}\right) \in Q^{n-1}} I_{q_{0}} \cdot \tau\left(\sigma_{1}\right)_{q_{0}, q_{1}} \cdot \ldots \cdot \tau\left(\sigma_{n-1}\right)_{q_{n-2}, q_{n-1}} \cdot \tau\left(\sigma_{n}\right)_{q_{n-1}, q_{n}} \\
& =\sum_{\left(q_{0}, \ldots, q_{n-1}\right) \in Q^{n}} I_{q_{0}} \cdot \tau\left(\sigma_{1}\right)_{q_{0}, q_{1}} \cdot \ldots \cdot \tau\left(\sigma_{n}\right)_{q_{n-1}, q_{n}} .
\end{aligned}
$$

Now we have

$$
\begin{aligned}
& \left(\llbracket M \rrbracket_{i}, w\right)=h_{\theta_{\tau}}(w) \cdot F=\sum_{q_{n} \in Q} h_{\theta_{\tau}}(w)_{q_{n}} \cdot F_{q_{n}} \\
& =\sum_{q_{n} \in Q} \sum_{\left(q_{0}, \ldots, q_{n-1}\right) \in Q^{n}} I_{q_{0}} \cdot \tau\left(\sigma_{1}\right)_{q_{0}, q_{1}} \cdot \ldots \cdot \tau\left(\sigma_{n}\right)_{q_{n-1}, q_{n}} \cdot F_{q_{n}}
\end{aligned}
$$

(by Equation (1) and right distributivity) $=\left(\llbracket M \rrbracket_{r}, w\right)$.

2. $\Rightarrow 1 .:$ Let $a, b, c \in A$. Let $\sigma \in \Sigma$. We construct the wfa $M=(Q, I, \tau, F)$ over $\Sigma$ and $A$ by $Q=\{p, q\}, I_{p}=a, I_{q}=b, F_{p}=c$, and $F_{q}$ can be chosen 
arbitrarily. Moreover, $\tau(\sigma)_{p, p}=\tau(\sigma)_{q, p}=1$ and $\tau(\sigma)_{p, q}=\tau(\sigma)_{q, q}=0$. Then $\left(\llbracket M \rrbracket_{i}, \sigma\right)=(a+b) \cdot c$ and $\left(\llbracket M \rrbracket_{r}, \sigma\right)=a c+b c$. Hence $(a+b) \cdot c=a c+b c$.

For semirings $A$, we obtain as consequence the following well-known fact.

Lemma 7. If $A$ is a semiring, then $\llbracket M \rrbracket_{i}=\llbracket M \rrbracket_{r}=\llbracket M \rrbracket_{f}$ for every wfa $M$ over $\Sigma$ and $A$.

Proof. Lemma 6 yields $\llbracket M \rrbracket_{i}=\llbracket M \rrbracket_{r}$. Moreover, $\llbracket M \rrbracket_{f}=\llbracket M \rrbracket_{i}$ follows from $\left(\llbracket M \rrbracket_{f}, w\right)=I \cdot h_{\Sigma}\left(\sigma_{1} \cdots \sigma_{n}\right) \cdot F=I \cdot \tau\left(\sigma_{1}\right) \cdot \ldots \cdot \tau\left(\sigma_{n}\right) \cdot F$ and Lemma 2.

Next we introduce deterministic and crisp automata.

Definition 8. Let $M=(Q, I, \tau, F)$ be a wfa over $\Sigma$ and $A$.

- We call $M$ deterministic if there is at most one $q \in Q$ with $I_{q} \neq 0$, and for every $\sigma \in \Sigma$ and $q \in Q$ there is at most one $q^{\prime} \in Q$ with $\tau(\sigma)_{q, q^{\prime}} \neq 0$.

- We call $M$ crisp if $I_{q} \in\{0,1\}$ and $\tau(\sigma)_{p, q} \in\{0,1\}$ for every $\sigma \in \Sigma$ and $p, q \in Q$.

- $M$ is crisp-deterministic, if $M$ is crisp and deterministic.

The following is easy to check.

Remark 9. For every deterministic wfa $M$ we have $\llbracket M \rrbracket_{i}=\llbracket M \rrbracket_{r}$, and if $M$ is crisp-deterministic, then $\operatorname{im}\left(\llbracket M \rrbracket_{i}\right)$ is finite.

Finally, we note that we obtain the classical concept of an automaton in our context as follows. A finite automaton (for short: $f s a$ ) over $\Sigma$ is a wfa $M$ over $\Sigma$ and the Boolean semiring $\mathbb{B}$. Clearly, then $\llbracket M \rrbracket_{i}=\llbracket M \rrbracket_{r}=\llbracket M \rrbracket_{f}$ by Lemma 7. The language recognized by $M$ is the set $L(M) \subseteq \Sigma^{*}$ defined by $L(M)=\left\{w \in \Sigma^{*} \mid\left(\llbracket M \rrbracket_{r}, w\right)=1\right\}$. A language $L \subseteq \Sigma^{*}$ is recognizable if there is an fsa $M$ over $\Sigma$ such that $L=L(M)$. As is well-known, for every recognizable language there is a crisp-deterministic fsa $M$ such that $L=L(M)$. Moreover, $M=(Q, I, \tau, F)$ can be chosen to be total, i.e. there is a state $q \in Q$ with $I_{q}=1$, and for every $\sigma \in \Sigma$ and $q \in Q$ there exists (a unique) $q^{\prime} \in Q$ with $\tau(\sigma)_{q, q^{\prime}}=1$. Furthermore, $M$ can be assumed to be co-accessible, i.e. for every $q \in F$ there is $w \in \Sigma^{*}$ with $h_{\theta_{\tau}}(w)_{q}=1$ (otherwise, reduce $F$ correspondingly).

Next we characterize series that are i-recognizable and r-recognizable by crisp-deterministic wfa, in terms of recognizable step functions. A series $\varphi \in$ $A\left\langle\left\langle\Sigma^{*}\right\rangle\right\rangle$ over $\Sigma$ and $A$ is a recognizable step function if there are $n \in \mathbb{N}$, recognizable languages $L_{1}, \ldots, L_{n} \subseteq \Sigma^{*}$, and $a_{1}, \ldots, a_{n} \in A$ such that $\varphi=\sum_{i=1}^{n} a_{i} \cdot \chi_{L_{i}}$.

Lemma 10. Let $\varphi \in A\left\langle\left\langle\Sigma^{*}\right\rangle\right\rangle$. Then $\varphi$ is a recognizable step function iff there exists a crisp-deterministic wfa $M$ over $\Sigma$ and $A$ such that $\varphi=\llbracket M \rrbracket_{i}=\llbracket M \rrbracket_{r}$. In particular, if $\varphi$ is a recognizable step function, then $\varphi$ is i-recognizable and r-recognizable.

Proof. " $\Rightarrow$ ": Let $n \in \mathbb{N}, L_{1}, \ldots, L_{n} \subseteq \Sigma^{*}$, and $a_{1}, \ldots, a_{n} \in A$ such that $L_{1}, \ldots, L_{n}$ are recognizable and $\varphi=\sum_{i=1}^{n} a_{i} \cdot \chi_{L_{i}}$. For every $i \in\{1, \ldots, n\}$, let $M_{i}=\left(Q_{i}, I_{i}, \tau_{i}, F_{i}\right)$ be a deterministic, total and co-accessible fsa over $\Sigma$ 
such that $L\left(M_{i}\right)=L_{i}$. We define the wfa $M=(Q, I, \tau, F)$ as follows: $Q=$ $Q_{1} \times \cdots \times Q_{n}$ and for every $\sigma \in \Sigma$ and $\left(q_{1}, \ldots, q_{n}\right),\left(q_{1}^{\prime} \ldots, q_{n}^{\prime}\right) \in Q$ :

$$
\begin{aligned}
I_{\left(q_{1}, \ldots, q_{n}\right)} & = \begin{cases}1, & \text { if }\left(I_{i}\right)_{q_{i}}=1 \text { for every } i \in\{1, \ldots, n\} ; \\
0, & \text { otherwise, }\end{cases} \\
\tau(\sigma)_{\left(q_{1}, \ldots, q_{n}\right),\left(q_{1}^{\prime}, \ldots, q_{n}^{\prime}\right)} & = \begin{cases}1, & \text { if } \tau_{i}(\sigma)_{q_{i}, q_{i}^{\prime}}=1 \text { for every } i \in\{1, \ldots, n\} ; \\
0, & \text { otherwise, }\end{cases} \\
F_{\left(q_{1}, \ldots, q_{n}\right)} & =\sum_{\substack{i \in\{1, \ldots, n\} \\
\left(F_{i}\right)_{q_{i}}=1}} a_{i} .
\end{aligned}
$$

Clearly, $M$ is crisp-deterministic. Let $w \in \Sigma^{*}$ and $\left(q_{1}, \ldots, q_{n}\right)$ be the unique state in $Q$ with $h_{\theta_{\tau}}(w)_{\left(q_{1}, \ldots, q_{n}\right)}=1$. Then $w \in L_{i}$ iff $\left(F_{i}\right)_{q_{i}}=1$ for every $i \in$ $\{1, \ldots, n\}$. Let $I_{w}=\left\{i \in\{1, \ldots, n\} \mid w \in L_{i}\right\}$. We obtain $(\varphi, w)=\sum_{i \in I_{w}} a_{i}=$ $F_{\left(q_{1}, \ldots, q_{n}\right)}=\left(\llbracket M \rrbracket_{i}, w\right)$, proving $\varphi=\llbracket M \rrbracket_{i}$. Remark 9 yields $\llbracket M \rrbracket_{i}=\llbracket M \rrbracket_{r}$.

" $\Leftarrow$ ": Let $M=(Q, I, \tau, F)$ be a crisp-deterministic wfa over $\Sigma$ and $A$ such that $\llbracket M \rrbracket_{i}=\llbracket M \rrbracket_{r}=\varphi$. By Remark $9, \operatorname{im}(\varphi)$ is finite. Let $a \in \operatorname{im}(\varphi)$. We show that $\varphi_{=a}$ is recognizable. We define an fsa $M_{a}^{\prime}=\left(Q, I^{\prime}, \tau^{\prime}, F_{a}^{\prime}\right)$ over $\Sigma$ by letting for every $p, q \in Q$ and $\sigma \in \Sigma: I_{q}^{\prime}=1$ iff $I_{q}=1 ; \tau^{\prime}(\sigma)_{p, q}=1$ iff $\tau(\sigma)_{p, q}=1$; and $\left(F_{a}^{\prime}\right)_{q}=1$ iff $F_{q}=a$. Then $L\left(M_{a}^{\prime}\right)=\varphi_{=a}$. Hence $\varphi=\sum_{a \in \operatorname{im}(\varphi)} a \cdot \chi_{\varphi=a}$ is a recognizable step function.

We finish this section with an easy characterization of recognizable step functions.

Proposition 11. Let $\varphi \in A\left\langle\left\langle\Sigma^{*}\right\rangle\right\rangle$. Then $\varphi$ is a recognizable step function iff $\operatorname{im}(\varphi)$ is finite and $\varphi_{=a}$ is recognizable for every $a \in A$.

Proof. Let $\varphi=\sum_{i=1}^{n} a_{i} \cdot \chi_{L_{i}}$ for some $n \in \mathbb{N}$, recognizable languages $L_{1}, \ldots, L_{n} \subseteq \Sigma^{*}$, and $a_{1}, \ldots, a_{n} \in A$. Clearly, $\operatorname{im}(\varphi)$ is finite. For every $I \subseteq\{1, \ldots, n\}$ we define a language $L_{I}^{\prime}$ as:

$$
L_{I}^{\prime}=\bigcap_{i \in I} L_{i} \cap \bigcap_{i \in\{1, \ldots, n\} \backslash I} \Sigma^{*} \backslash L_{i} .
$$

It follows from the closure properties of the class of recognizable languages that every $L_{I}^{\prime}$ is recognizable. Choose $a \in A$ and let $\mathcal{I}_{a}=\left\{I \subseteq\{1, \ldots, n\} \mid \sum_{i \in I} a_{i}=\right.$ $a\}$. Then $L=\bigcup_{I \in \mathcal{I}_{a}} L_{I}^{\prime}$ is a recognizable language. Now let $w \in \Sigma^{*}$ and put $I_{w}=\left\{i \in\{1, \ldots, n\} \mid w \in L_{i}\right\}$. Then $w \in \varphi_{=a}$ iff $I_{w} \in \mathcal{I}_{a}$ iff $w \in L$ Hence, $\varphi=a=L$.

For the converse, note that $\varphi=\sum_{a \in \operatorname{im}(\varphi)} a \cdot \chi_{\varphi_{=a}}$ which is a recognizable step function by assumption.

\section{Recognizable series and determinizability}

In this section, we will investigate the relationships between i-recognizable series, r-recognizable series, and recognizable step functions. We also consider conditions under which for every wfa an i-equivalent crisp-deterministic one exists.

We let $\mathcal{N}=(\mathbb{N},+, \cdot, 0,1)$ be the semiring of natural numbers with the usual addition and multiplication. We will use the following lemma. 
Lemma 12 ( [5], Cor. III.2.4,2.5). Let $\rho: \Sigma^{*} \rightarrow \mathbb{N}$ be an r-recognizable series over $\Sigma$ and $\mathcal{N}$. Then for all $c, t \in \mathbb{N}$, the languages $\rho^{-1}(t)$ and $\rho^{-1}(t+c \mathbb{N})$ are recognizable.

We call $A$ additively locally finite (multiplicatively locally finite, respectively) if for every finite $B \subseteq A$, the smallest sub-monoid of $(A,+, 0)$ (of $(A, \cdot, 1)$, respectively) containing $B$ is finite. Now we will show:

Theorem 13. Let $A$ be additively and multiplicatively locally finite, and let $\varphi \in A\left\langle\left\langle\Sigma^{*}\right\rangle\right\rangle$ be r-recognizable. Then $\varphi$ is a recognizable step function.

Proof. Let $M=(Q, I, \tau, F)$ be a wfa with $\llbracket M \rrbracket_{r}=\varphi$. We define $B=$ $\left\{I_{q}, \tau(\sigma)_{p, q}, F_{q} \mid p, q \in Q, \sigma \in \Sigma\right\}$. Let $Y$ comprise all finite products of elements from $B$, and let $K$ consist of all finite sums of elements from $Y$. By assumption, $K$ is finite. Hence $\operatorname{im}(\varphi)$ is also finite. It only remains to show that $\varphi=x$ is a recognizable language, for each $x \in K$.

Note that weight $_{M}(P, w) \in Y$ for every $w=\sigma_{1} \cdots \sigma_{n} \in \Sigma^{*}$ and $P \in Q^{n+1}$. For each $a \in Y$ we define a crisp wfa $M_{a}^{\prime}=\left(Q^{\prime}, I^{\prime}, \tau^{\prime}, F_{a}^{\prime}\right)$ over $\Sigma$ and $\mathcal{N}$ as follows: $Q^{\prime}=Q \times Y$ and for every $\sigma \in \Sigma$ and $(q, y),\left(q^{\prime}, y^{\prime}\right) \in Q^{\prime}$, let

$$
\begin{aligned}
\tau^{\prime}(\sigma)_{(q, y),\left(q^{\prime}, y^{\prime}\right)} & = \begin{cases}1, & \text { if } y^{\prime}=y \cdot \tau(\sigma)_{q, q^{\prime}} \\
0, & \text { otherwise, }\end{cases} \\
I_{(q, y)}^{\prime} & =\left\{\begin{array}{ll}
1, & \text { if } y=I_{q} ; \\
0, & \text { otherwise, }
\end{array} \text { and }\left(F_{a}^{\prime}\right)_{(q, y)}= \begin{cases}1, & \text { if } y \cdot F_{q}=a ; \\
0, & \text { otherwise. }\end{cases} \right.
\end{aligned}
$$

Let $w=\sigma_{1} \cdots \sigma_{n} \in \Sigma^{*}$ and $a \in Y$. We observe that for every path $P=$ $\left(q_{0}, \ldots, q_{n}\right) \in Q^{n+1}$ with weight $_{M}(P, w)=a$ there is a unique path $P^{\prime}=$

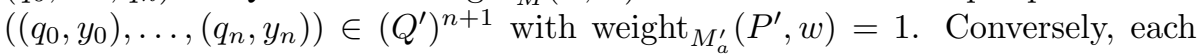
path $P^{\prime \prime} \in\left(Q^{\prime}\right)^{n+1}$ with non-zero weight in $M_{a}^{\prime}$ for $w$ arises in this form. It follows that $\left(\llbracket M_{a}^{\prime} \rrbracket_{r}, w\right) \in \mathbb{N}$ is precisely the number of all paths $P$ in $Q^{n+1}$ with weight $_{M}(P, w)=a$. Consequently, in $A$ we have

$$
(\varphi, w)=\left(\llbracket M \rrbracket_{r}, w\right)=\sum_{P \in Q^{n+1}} \operatorname{weight}_{M}(P, w)=\sum_{a \in Y}\left(\llbracket M_{a}^{\prime} \rrbracket_{r}, w\right) a,
$$

where, for every $m \in \mathbb{N}$, we write $m a$ as a shorthand for $a+a+\cdots+a(m$ summands). Next we use an argument similar to one used in [9], proof of Prop. 6.3. For each $a \in Y$, the cyclic submonoid $\langle a\rangle$ of $(A,+, 0)$ is finite. Choose a minimal $m_{a} \in \mathbb{N}$ such that $m_{a} a=\left(m_{a}+y\right) a$ for some $y>0$, and let $c_{a}$ be the smallest such $y>0$. We put $d_{a}=m_{a}+c_{a}-1$; note that then $d_{0}=0$. Then $\langle a\rangle=\left\{0, a, 2 a, \ldots, d_{a} a\right\}$. So for each $s \in \mathbb{N}$ there is a uniquely determined $t \in\left\{0, \ldots, d_{a}\right\}$ such that $s a=t a$. Note that if $0 \leq t<m_{a}$, then $s a=t a$ iff $s=t$, and if $m_{a} \leq t \leq d_{a}$, then $s a=t a$ iff $s \in t+c_{a} \mathbb{N}$. Now let $L_{a, t}=\left\{w \in \Sigma^{*} \mid\left(\llbracket M_{a}^{\prime} \rrbracket_{r}, w\right) a=t a\right\}$, for each $t \in \mathbb{N}$ with $0 \leq t \leq d_{a}$; note that $L_{0,0}=\Sigma^{*}$. We claim that $L_{a, t}$ is recognizable. We have

$$
\begin{aligned}
& \text { - } L_{a, t}=\left\{w \in \Sigma^{*} \mid\left(\llbracket M_{a}^{\prime} \rrbracket_{r}, w\right)=t\right\} \text { if } 0 \leq t<m_{a} \text {, and } \\
& \text { - } L_{a, t}=\left\{w \in \Sigma^{*} \mid\left(\llbracket M_{a}^{\prime} \rrbracket_{r}, w\right) \in t+c_{a} \mathbb{N}\right\} \text { if } m_{a} \leq t \leq d_{a} .
\end{aligned}
$$


In each case, $L_{a, t}$ is recognizable by Lemma 12 .

Now let $x \in K$. We wish to show that $\varphi_{=x}$ is recognizable. Let $w \in \Sigma^{*}$. By the above, for every $a \in Y$ there is a unique number $t_{a} \in\left\{0, \ldots, d_{a}\right\}$ with $\left(\llbracket M_{a}^{\prime} \rrbracket_{r}, w\right) a=t_{a} a$, and so $w \in L_{a, t_{a}}$. Let $\mathcal{Y}$ be the set of all mappings $F: Y \rightarrow \mathbb{N}$ such that $x=\sum_{a \in Y} F(a) a$ and $F(a) \in\left\{0, \ldots, d_{a}\right\}$ for every $a \in Y$. Hence, by $(\star)$, we have $w \in \varphi=x$ iff $x=\sum_{a \in Y}\left(\llbracket M_{a}^{\prime} \rrbracket_{r}, w\right) a$ iff there is an $F \in \mathcal{Y}$ with $w \in L_{a, F(a)}$ for every $a \in Y$, thus:

$$
\varphi_{=x}=\bigcup_{F \in \mathcal{Y}} \bigcap_{a \in Y} L_{a, F(a)},
$$

which is a recognizable language because both $Y$ and $\mathcal{Y}$ are finite.

Conversely, assuming that the image of every recognizable series over $A$ is finite, we can deduce that $A$ is additively and multiplicatively locally finite. Note that here our alphabet $\Sigma$ is fixed.

Lemma 14. Let $|\Sigma| \geq 2$. If for every wfa $M$ over $\Sigma$ and $A, \operatorname{im}\left(\llbracket M \rrbracket_{i}\right)$ or $\operatorname{im}\left(\llbracket M \rrbracket_{r}\right)$ is finite, then $A$ is additively and multiplicatively locally finite.

Proof. We show that the additive monoid $(A,+, 0)$ and the multiplicative monoid $(A, \cdot, 1)$ are locally finite.

For the additive monoid it suffices to show that for every $a \in A$ the cyclic submonoid of $(A,+, 0)$ generated by $a$ is finite because + is commutative and associative. Let $a \in A$ and construct the wfa $M=(\{p, q\}, I, \tau, F)$ with $I_{p}=1$, $I_{q}=F_{p}=0$, and $F_{q}=a$. Moreover, for every $\sigma \in \Sigma$ we define $\tau(\sigma)_{p, p}=$ $\tau(\sigma)_{p, q}=\tau(\sigma)_{q, q}=1$, and $\tau(\sigma)_{q, p}=0$. Then for every $\sigma \in \Sigma$ and $n \in \mathbb{N}$ we have $\left(\llbracket M \rrbracket_{i}, \sigma^{n}\right)=\left(\llbracket M \rrbracket_{r}, \sigma^{n}\right)=a+\cdots+a(n$ times $)$. Thus, the finite set $\operatorname{im}\left(\llbracket M \rrbracket_{i}\right) \cap \operatorname{im}(\llbracket M \rrbracket)_{r}$ contains the cyclic submonoid of $(A,+, 0)$ generated by $a$.

Next we show that the multiplicative monoid is locally finite. Let $n \in \mathbb{N}$ and $a_{1}, \ldots, a_{n} \in A$. We show that the set $A^{\prime}=\left\{a_{l_{1}} \cdots a_{l_{k}} \mid k \in \mathbb{N}, l_{1}, \ldots, l_{k} \in\right.$ $\{1, \ldots, n\}\}$ is finite. Let $\sigma_{1}, \sigma_{2} \in \Sigma$ be distinct symbols. We construct a wfa $M^{\prime}=\left(Q^{\prime}, I^{\prime}, \tau^{\prime}, F^{\prime}\right)$ over $\Sigma$ and $A$ with $Q^{\prime}=\left\{q_{0}, q_{1}, \ldots, q_{n}\right\}, I_{q_{0}}^{\prime}=F_{q_{0}}^{\prime}=1$ and $I_{q}^{\prime}=F_{q}^{\prime}=0$ for every $q \in Q^{\prime} \backslash\left\{q_{0}\right\}$, and $\tau^{\prime}$ is defined as follows (see Figure 2):

- $\tau^{\prime}\left(\sigma_{1}\right)_{q_{i-1}, q_{i}}=1$ for every $i \in\{1, \ldots, n\}$,

- $\tau^{\prime}\left(\sigma_{2}\right)_{q_{i}, q_{0}}=a_{i}$ for every $i \in\{1, \ldots, n\}$, and

- $\tau^{\prime}(\sigma)_{q, q^{\prime}}=0$ for every other combination of $\sigma \in \Sigma$ and $q, q^{\prime} \in Q^{\prime}$.

Then $\left(\llbracket M^{\prime} \rrbracket_{i}, \sigma_{1}^{l_{1}} \sigma_{2} \sigma_{1}^{l_{2}} \sigma_{2} \cdots \sigma_{1}^{l_{k}} \sigma_{2}\right)=\left(\llbracket M^{\prime} \rrbracket_{r}, \sigma_{1}^{l_{1}} \sigma_{2} \sigma_{1}^{l_{2}} \sigma_{2} \cdots \sigma_{1}^{l_{k}} \sigma_{2}\right)=a_{l_{1}} \cdots a_{l_{k}}$ for every $k \in \mathbb{N}$ and $l_{1}, \ldots, l_{k} \in\{1, \ldots, n\}$. Thus, $A^{\prime} \subseteq \operatorname{im}\left(\llbracket M^{\prime} \rrbracket_{i}\right) \cap \operatorname{im}\left(\llbracket M^{\prime} \rrbracket_{r}\right)$, and therefore $A^{\prime}$ is finite.

In fact, Lemma 14 generalizes the following results of [15, 23].

Corollary 15. If for every wfa over $A$ there is an i-equivalent crispdeterministic wfa over $A$, then $A$ is locally finite provided that one of the following conditions hold.

1. A is a lattice-ordered monoid (cf. only-if part of Theorem 3.4 of [23]). 


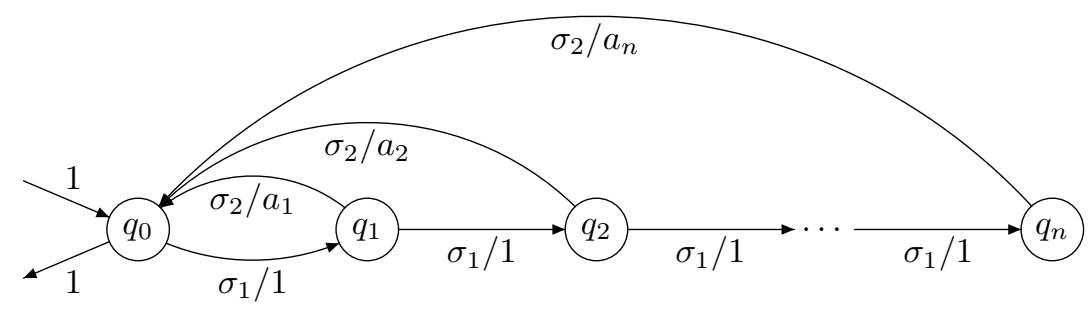

Figure 2: wfa $M^{\prime}$ from the proof of Lemma 14.

2. A is the semiring-reduct of a residuated lattice (cf. only-if part of Theorem 4.2 of [15]).

Proof. Since both, lattice-ordered monoids and semiring-reducts of residuated lattices are particular strong bimonoids, the two statements follow from Remark 9 and Lemma 14.

The following summarizes our results.

Theorem 16. Let $|\Sigma| \geq 2$. Then the following two statements are equivalent:

1. For every wfa $M$ over $\Sigma$ and $A$ there is an r-equivalent crisp-deterministic wfa $M^{\prime}$ over $\Sigma$ and $A$.

2. A is additively and multiplicatively locally finite.

Proof. (1) $\Rightarrow(2)$ : This follows from Remark 9 and Lemma 14.

$(2) \Rightarrow(1)$ : Immediate by Theorem 13 and Lemma 10.

Next we will investigate properties of i-recognizable series. The idea of the following lemma is similar to Lemma 3.10 of [12]. It will be a useful tool for constructing weighted automata from finite pointed $\Sigma$-algebras.

Lemma 17. Let $(P, \theta, q)$ be a finite pointed $\Sigma$-algebra.

1. For every mapping $f: P \rightarrow A$ there is a crisp-deterministic wfa $M$ over $\Sigma$ and $A$ such that $\llbracket M \rrbracket_{i}=h_{\theta} ; f$.

2. For every $F \subseteq P$ the language $h_{\theta}^{-1}(F) \subseteq \Sigma^{*}$ is recognizable.

Proof. First we prove Statement 1 . We define $M=(P, I, \tau, F)$ as follows for every $\sigma \in \Sigma$ and $p, p^{\prime} \in P$ :

$$
\begin{aligned}
I_{p} & =\left\{\begin{array}{ll}
1, & \text { if } p=q ; \\
0, & \text { otherwise },
\end{array} \quad \tau(\sigma)_{p, p^{\prime}}= \begin{cases}1, & \text { if } p^{\prime}=\theta(\sigma)(p) ; \\
0, & \text { otherwise }\end{cases} \right. \\
F_{p} & =f(p) .
\end{aligned}
$$

Observe that $M$ is crisp-deterministic. It is easy to check that for every $w \in \Sigma^{*}$ and $p \in P$ we have $h_{\theta_{\tau}}(w)_{p}=1$ if $h_{\theta}(w)=p$ and $h_{\theta_{\tau}}(w)_{p}=0$ otherwise. Thus, for every $w \in \Sigma^{*}$ we have $\left(\llbracket M \rrbracket_{i}, w\right)=h_{\theta_{\tau}}(w) \cdot F=\sum_{p \in P} h_{\theta_{\tau}}(w)_{p} \cdot F_{p}=$ $F_{h_{\theta}(w)}=f\left(h_{\theta}(w)\right)=\left(h_{\theta} ; f\right)(w)$.

Next we prove Statement 2. Let $f: P \rightarrow \mathbb{B}$ be defined for every $p \in P$ by $f(p)=1$ iff $p \in F$. By Statement 1 there is an fsa $M$ over $\Sigma$ with $\llbracket M \rrbracket_{i}=h_{\theta} ; f$. This yields $L(M)=\left\{w \in \Sigma^{*} \mid f\left(h_{\theta}(w)\right)=1\right\}=h_{\theta}^{-1}(F)$. 
For our subsequent results, we will need particular finiteness conditions on the strong bimonoid $A$ (which may be infinite), which we introduce next.

Definition 18. For every $B \subseteq A$ the weak closure of $B$, denoted by $\operatorname{cl}(B)$, is the smallest subset $C \subseteq A$ such that $B \subseteq C$ and $c+c^{\prime} \in C$ and $c \cdot b \in C$ for all $b \in B$ and $c, c^{\prime} \in C$. We say that $A$ is weakly locally finite if $\operatorname{cl}(B)$ is finite for every finite subset $B \subseteq A$. Moreover, we say that $A$ is locally finite if, for every finite $B \subseteq A$, the smallest sub-bimonoid of $A$ containing $B$ is finite.

Trivially, if $A$ is locally finite, then $A$ is weakly locally finite, and if $A$ is weakly locally finite, then $A$ is additively and multiplicatively locally finite. For example, if $A$ is a bounded lattice, then $A$ is a stong bimonoid which is additively and multiplicatively locally finite, but need not be locally finite. The following is easy to check.

Remark 19. If $A$ is right distributive, then $A$ is additively and multiplicatively locally finite iff $A$ is weakly locally finite. If $A$ is left distributive, then $A$ is weakly locally finite iff $A$ is locally finite. In particular, if $A$ is a semiring, then $A$ is additively and multiplicatively locally finite iff $A$ is locally finite.

The idea of the following lemma is based on Lemma 3.14 of [12].

Lemma 20. Let $\varphi \in A\left\langle\left\langle\Sigma^{*}\right\rangle\right\rangle$ be i-recognizable. If $A$ is weakly locally finite, then there is a finite pointed $\Sigma$-algebra $(P, \theta, q)$ and a mapping $f: P \rightarrow A$ such that $\varphi=h_{\theta} ; f$.

Proof. Choose a wfa $M=(Q, I, \tau, F)$ over $\Sigma$ and $A$ such that $\varphi=\llbracket M \rrbracket_{i}$. Let $B=\left\{I_{q}, \tau(\sigma)_{p, q} \mid p, q \in Q, \sigma \in \Sigma\right\}$. By assumption, the weak closure $\operatorname{cl}(B)$ is finite. Then $P=\left\{h_{\theta_{\tau}}(w) \mid w \in \Sigma^{*}\right\} \subseteq \operatorname{cl}(B)^{Q}$ is also finite, and $\left(P, \theta_{\tau}, I\right)$ is a pointed $\Sigma$-algebra. Moreover, we define the mapping $f$ by $f(v)=v \cdot F$ for every $v \in P$. Then $\left(\llbracket M \rrbracket_{i}, w\right)=h_{\theta_{\tau}}(w) \cdot F=f\left(h_{\theta_{\tau}}(w)\right)=\left(h_{\theta_{\tau}} ; f\right)(w)$.

The following result summarizes three important properties of i-recognizable series if $A$ is weakly locally finite.

Theorem 21. Let $A$ be weakly locally finite and $\varphi \in A\left\langle\left\langle\Sigma^{*}\right\rangle\right\rangle$ be i-recognizable.

1. For any $E \subseteq A$ the language $\varphi^{-1}(E)$ is recognizable. In particular, $\varphi$ is a recognizable step function.

2. For any mapping $g: A \rightarrow A$, the series $\varphi ; g$ is again i-recognizable.

3. (Determinization) There exists a crisp-deterministic wfa $M$ over $\Sigma$ and A such that $\varphi=\llbracket M \rrbracket_{i}$.

Proof. By Lemma 20 there are a finite pointed $\Sigma$-algebra $(P, \theta, q)$ and a mapping $f: P \rightarrow A$ such that $\varphi=h_{\theta} ; f$. First we show Statment 1. Since $\varphi^{-1}(E)=h_{\theta}^{-1}\left(f^{-1}(E)\right)$ and $f^{-1}(E) \subseteq P$, it follows from Lemma 17(2) that $\varphi^{-1}(E)$ is recognizable. In particular, for every $a \in A$, the language $\varphi_{=a}$ is recognizable. Moreover, $\operatorname{im}(\varphi) \subseteq f(P)$ is finite. Hence, $\varphi=\sum_{a \in \operatorname{im}(\varphi)} a \cdot \chi_{\varphi_{=a}}$ is a recognizable step function.

Next we show Statements 2 and 3. Let $g: A \rightarrow A$. We have $\varphi ; g=$ $\left(h_{\theta} ; f\right) ; g=h_{\theta} ;(f ; g)$. Then by Lemma $17(1)$ there is a crisp-deterministic wfa $M$ over $\Sigma$ and $A$ with $\varphi ; g=\llbracket M \rrbracket_{i}$. This proves Statement 2 and also Statement 3 (let $g$ be the identity mapping). 
We note that part 3 of Theorem 21 would also follow from part 1 and Lemma 10, but the present argument is simpler and leads to automata with smaller state sets.

Theorem 21(3) generalizes the following results of [3,24].

Corollary 22. For every $x$-recognizable series $\varphi \in A\left\langle\left\langle\Sigma^{*}\right\rangle\right\rangle$ there is a crispdeterministic wfa $M$ such that $\llbracket M \rrbracket_{x}=\varphi$ assuming that one of the following conditions holds.

1. A is a bounded, complete, distibutive lattice and $x=f$ (cf. Theorem 2.1 of [3]).

2. A is a bounded, distributive lattice and $x=r$ (cf. Theorem 2.1 of [24]).

Proof. 1. In [3] Bělohlávek defined recognizable series by means of wfa over bounded, complete, locally finite lattices with the free monoid semantics; in fact, he also needed that the lattices are distributive [4]; then locally finiteness follows from boundedness and distributivity. Now let $\varphi \in A\left\langle\left\langle\Sigma^{*}\right\rangle\right\rangle$ be recognizable in this sense. Since every bounded, complete, distibutive lattice is a locally finite semiring, it follows from Lemma 7 that $\varphi$ is $i$-recognizable. Since every locally finite semiring is a weakly locally finite strong bimonoid, Theorem 21(3) shows Statement 1.

Proof of 2: In [24] Li and Pedrycz defined recognizable series by means of wfa over bounded, distributive lattices with the run semantics. The proof of Statement 2 uses the same arguments as the proof of Statement 1.

Now we can give an analogue of Theorem 16 for the i-behavior of wfa.

Theorem 23. Let $|\Sigma| \geq 2$ and let $A$ be right distributive. Then the following two statements are equivalent:

1. For every wfa $M$ over $\Sigma$ and $A$ there is an i-equivalent crisp-deterministic wfa $M^{\prime}$ over $\Sigma$ and $A$.

2. A is additively and multiplicatively locally finite.

Proof. This theorem is an immediate consequence of Remark 9, Lemma 14, Remark 19, and Theorem 21(3).

The following summarizes our results.

Theorem 24. Let $A$ be additively and multiplicatively locally finite, and let $\varphi \in A\left\langle\left\langle\Sigma^{*}\right\rangle\right\rangle$. Then the following are equivalent:

1. $\varphi$ is r-recognizable.

2. $\varphi$ is a recognizable step function.

Moreover, if $A$ is weakly locally finite, these conditions are equivalent to

3. $\varphi$ is i-recognizable.

Proof. This is an immediate consequence of Lemma 10 and Theorems 13 and 21(1). 
Let $\leq$ be a partial order on $A$. Moreover, let $\varphi \in A\left\langle\left\langle\Sigma^{*}\right\rangle\right\rangle$ be a series and $a \in A$. Then the $a$-cut of $\varphi$ is the set $\varphi \geq a=\left\{w \in \Sigma^{*} \mid(\varphi, w) \geq a\right\}$. Several authors $[6,17,31]$ have investigated these cuts of $\varphi$. The following is straightforward, but together with Theorem 24 it provides the connection to the work cited.

Lemma 25. Let $\leq$ be a partial order on $A$, and let $\varphi \in A\left\langle\left\langle\Sigma^{*}\right\rangle\right\rangle$. The following statements are equivalent.

1. $\varphi$ is a recognizable step function.

2. $\operatorname{im}(\varphi)$ is finite and $\varphi_{\geq a}$ is recognizable for every $a \in \operatorname{im}(\varphi)$.

Proof. First we prove $(1) \Rightarrow(2)$. Clearly, $\operatorname{im}(\varphi)$ is finite. By Proposition 11 we have that $\varphi_{=b}$ is recognizable for every $b \in \operatorname{im}(\varphi)$. Let $a \in \operatorname{im}(\varphi)$. Then

$$
\varphi \geq a=\bigcup_{b \in \operatorname{im}(\varphi)} \varphi=b
$$

which is a finite union of recognizable languages and, hence, recognizable.

$(2) \Rightarrow(1)$. Since $\varphi=\sum_{a \in \operatorname{im}(\varphi)} a \cdot \chi_{\varphi=a}$, it suffices to show that $\varphi_{=a}$ is recognizable for every $a \in \operatorname{im}(\varphi)$. But

$$
\varphi=a=\varphi_{\geq a} \backslash \bigcup_{b \in \operatorname{im}(\varphi)} \varphi_{\geq \geq b},
$$

which implies our claim.

Now we can show that we have generalized a result of $[22,24]$.

Corollary 26. For every $\varphi \in A\left\langle\left\langle\Sigma^{*}\right\rangle\right\rangle$ the following conditions are equivalent

(i) $\varphi$ is x-recognizable.

(ii) $\operatorname{im}(\varphi)$ is finite and $\varphi_{=a} \in \Sigma^{*}$ is recognizable for every $a \in A$.

(iii) $\operatorname{im}(\varphi)$ is finite and $\varphi_{\geq a} \in \Sigma^{*}$ is recognizable for every $a \in A$.

provided that one of the following properties holds.

1. A is a muliplicatively locally finite, commutative, strong bimonoid of the form $([0,1], \max , *, 0,1)$ where $*$ is a $t$-norm, and $x=i$ (cf. Theorem 2.6 (2)-(4) of [22]).

2. A is a bounded, distributive lattice, and $x=r$ (cf. Theorem 2.2 of [24]).

Proof. 1. It is easy to prove that a t-norm [17] distributes from left and right over max. Since $A=([0,1], \max , *, 0,1)$ is distributive, additively locally finite and, by assumption, also multiplicatively locally finite, it follows from Remark 19 , that $A$ is a locally finite. Hence, in Theorem 2.6 of [24], Li and Pedrycz considered particular locally finite, commutative semirings. Then the stated equivalence under Property 1 follows from Theorem 24 and Lemma 25.

2. Since every bounded, distributive lattice is a locally finite semiring, the statement follows from Lemma 7, Theorem 24, and Lemma 25. 
Finally we show that for arbitrary strong bimonoids $A$, the concepts of irecognizability and r-recognizability differ. Clearly, by Lemma 6 and Theorem 24, $A$ should neither be right distributive nor weakly locally finite. We will give two examples. In the first example we construct an i-recognizable series that is not r-recognizable. In the second example we consider an r-recognizable series that is not i-recognizable.

Example 27. We consider the set $\mathbb{N}$ of natural numbers and we define two new commutative operations $\oplus$ and $\odot$ on $\mathbb{N}$ as follows. First, let $0 \oplus a=a, 0 \odot a=0$, and $1 \odot a=a$ for every $a \in \mathbb{N}$. If $a, b \in \mathbb{N} \backslash\{0\}$ with $a \leq b$, we put (with + being the usual addition on $\mathbb{N}$ )

$$
a \oplus b= \begin{cases}b, & \text { if } b \text { is even; } \\ b+1, & \text { if } b \text { is odd }\end{cases}
$$

If $a, b \in \mathbb{N} \backslash\{0,1\}$ with $a \leq b$, let

$$
a \odot b= \begin{cases}b+1, & \text { if } b \text { is even; } \\ b, & \text { if } b \text { is odd. }\end{cases}
$$

Then $A=(\mathbb{N}, \oplus, \odot, 0,1)$ is a strong bimonoid. Note that $A$ is neither right distributive (e.g., $5=(3 \oplus 3) \odot 2 \neq(3 \odot 2) \oplus(3 \odot 2)=4)$ nor weakly locally finite (e.g., $a+1 \in\{a \oplus a, a \odot a\}$, and hence $a \in\langle 1\rangle$ for every $a \in \mathbb{N}$ ). Now consider the wfa $M=(Q, I, \tau, F)$ with two states and $I_{p}=\tau(\sigma)_{p, q}=F_{q}=2$ for every $\sigma \in \Sigma$ and $p, q \in Q$. Then $\left(\llbracket M \rrbracket_{i}, \sigma^{n}\right)=2 n+4$ for every $\sigma \in \Sigma$ and $n \in \mathbb{N}$, hence, $\operatorname{im}\left(\llbracket M \rrbracket_{i}\right)$ is infinite.

On the other hand we claim that for each wfa $M^{\prime}=\left(Q^{\prime}, I^{\prime}, \tau^{\prime}, F^{\prime}\right)$ over $\Sigma$ and $A$, the set $\operatorname{im}\left(\llbracket M^{\prime} \rrbracket_{r}\right)$ is finite. Indeed, let $m=\max \left\{I_{p}^{\prime}, \tau^{\prime}(\sigma)_{p, q}, F_{q}^{\prime} \mid\right.$ $\left.p, q \in Q^{\prime}, \sigma \in \Sigma\right\} \in \mathbb{N}$. Then the definition of the r-behavior of $M$ shows that $\left(\llbracket M^{\prime} \rrbracket_{r}, w\right) \leq m+2$ for each $w \in \Sigma^{*}$, which yields our claim. Hence, the series $\llbracket M \rrbracket_{i}$ is not r-recognizable.

Example 28. We define two binary commutative operations $\oplus$ and $\odot$ on the set $\mathbb{N}^{2}$ of pairs of natural numbers as follows. First, let $(0,0) \oplus(a, b)=(a, b)$, $(0,0) \odot(a, b)=(0,0)$, and $(1,0) \odot(a, b)=(a, b)$ for every $(a, b) \in \mathbb{N}^{2}$. If $(a, b),\left(a^{\prime}, b^{\prime}\right) \in \mathbb{N}^{2} \backslash\{(0,0)\}$, we put

$$
(a, b) \oplus\left(a^{\prime}, b^{\prime}\right)= \begin{cases}\left(a, b+b^{\prime}\right), & \text { if } a=a^{\prime} \\ \left(1, b+b^{\prime}\right), & \text { otherwise }\end{cases}
$$

If $(a, b),\left(a^{\prime}, b^{\prime}\right) \in \mathbb{N}^{2} \backslash\{(0,0),(1,0)\}$, let

$$
(a, b) \odot\left(a^{\prime}, b^{\prime}\right)= \begin{cases}\left(a \cdot a^{\prime}, 1\right), & \text { if } b=b^{\prime}=1 \\ (1,2), & \text { otherwise }\end{cases}
$$

Then $A=\left(\mathbb{N}^{2}, \oplus, \odot,(0,0),(1,0)\right)$ is a strong bimonoid. Now consider the wfa $M=(Q, I, \tau, F)$ with two states and $I_{p}=\tau(\sigma)_{p, q}=F_{q}=(2,1)$ for every $\sigma \in \Sigma$ and $p, q \in Q$. Then $\left(\llbracket M \rrbracket_{r}, \sigma^{n}\right)=\left(2^{n+2}, 2^{n+1}\right)$ for every $\sigma \in \Sigma$ and $n \in \mathbb{N}$.

We claim that $\llbracket M \rrbracket_{r}$ is not i-recognizable. Let $M^{\prime}=\left(Q^{\prime}, I^{\prime}, \tau^{\prime}, F^{\prime}\right)$ be a wfa over $\Sigma$ and $A$. For each $\left(a, a^{\prime}\right) \in \mathbb{N}^{2}$ we let $\pi_{1}\left(a, a^{\prime}\right)=a$ and $\pi_{2}\left(a, a^{\prime}\right)=a^{\prime}$. If 
$|Q|=1$, then $\pi_{2}\left(\llbracket M^{\prime} \rrbracket_{i}, w\right) \leq \max \left\{2, \pi_{2}\left(I_{p}^{\prime}\right), \pi_{2}\left(\tau^{\prime}(\sigma)_{p, q}\right), \pi_{2}\left(F_{q}^{\prime}\right) \mid p, q \in Q^{\prime}, \sigma \in\right.$ $\Sigma\}$ for every $w \in \Sigma^{*}$, which proves that $\llbracket M \rrbracket_{r} \neq \llbracket M^{\prime} \rrbracket_{i}$. Now assume that $|Q|>$ 1. Let $m=\max \left\{\pi_{1}\left(I_{p}^{\prime}\right) \mid p \in Q^{\prime}\right\}$ and $m^{\prime}=m \cdot \max \left\{\pi_{1}\left(\tau^{\prime}(\sigma)_{p, q}\right) \mid p, q \in Q^{\prime}, \sigma \in\right.$ $\Sigma$ \}. It is easy to show by induction on the length of $w$ that for every $w \in \Sigma^{*}$ and $p \in Q^{\prime}$ we have that $\pi_{1}\left(h_{\theta_{\tau^{\prime}}}(w)_{p}\right)>m$ implies both $\pi_{1}\left(h_{\theta_{\tau^{\prime}}}(w)_{p}\right) \leq m^{\prime}$ and $\pi_{2}\left(h_{\theta_{\tau^{\prime}}}(w)_{p}\right)>1$. This implies $\pi_{1}\left(\llbracket M^{\prime} \rrbracket_{i}, w\right) \leq m^{\prime} \cdot \max \left\{\pi_{1}\left(F_{q}^{\prime}\right) \mid q \in Q^{\prime}\right\}$ for every $w \in \Sigma^{*}$. Hence, $\llbracket M \rrbracket_{r} \neq \llbracket M^{\prime} \rrbracket_{i}$.

Acknowledgments: The authors are grateful to Andreja Tepavčević and Branimir Šešelja for helpful discussions and valuable suggestions which improved the paper.

\section{References}

[1] J. Albert and J. Kari. Digital image compression. In M. Droste, W. Kuich, and H. Vogler, editors, Handbook of Weighted Automata, chapter 11. Springer-Verlag, 2009. to appear.

[2] Chr. Baier, F. Ciesinsky, and M. Grösser. Model checking of distributed randomized systems. In M. Droste, W. Kuich, and H. Vogler, editors, Handbook of Weighted Automata, chapter 13. Springer-Verlag, 2009. to appear.

[3] R. Bělohlávek. Determinism and fuzzy automata. Inform. Sci., 143:205209, 2002.

[4] R. Bělohlávek. personal communication. 2008.

[5] J. Berstel and Ch. Reutenauer. Rational Series and Their Languages, volume 12 of EATCS-Monographs. Springer-Verlag, 1988.

[6] B. Borchardt, A. Maletti, B. Šešelja, A. Tepavčevic, and H. Vogler. Cut sets as recognizable tree languages. Fuzzy Sets and Systems, 157:1560-1571, 2006 .

[7] A. L. Buchsbaum, R. Giancarlo, and J. R. Westbrook. On the determinization of weighted finite automata. SIAM Journal on Computing, 30(5):15021531, 2000.

[8] K. Culik and J. Kari. Image compression using weighted finite automata. Computer and Graphics, 17:305-313, 1993.

[9] M. Droste and P. Gastin. Weighted automata and weighted logics. Theor. Comput. Sci., 380(1-2):69-86, 2007.

[10] S. Eilenberg. Automata, Languages, and Machines - Volume A, volume 59 of Pure and Applied Mathematics. Academic Press, 1974.

[11] M. Eramian. Image Texture Analysis Using Weighted Finite Automata. $\mathrm{PhD}$ thesis, University of Western Ontario, London, Ontario, 2002.

[12] Z. Fülöp and H. Vogler. Weighted tree automata and tree transducers. In M. Droste, W. Kuich, and H. Vogler, editors, Handbook of Weighted Automata, chapter 9. Springer-Verlag, 2009. to appear. 
[13] G. Grätzer. General Lattice Theory. Birkhäuser Verlag, 2nd edition, 1979.

[14] U. Hafner. Low Bit-Rate Image and Video Coding with Weighted Finite Automata. PhD thesis, Universität Würzburg, Germany, 1999.

[15] J. Ignjatović, M. Ćirić, and S. Bogdanović. Determinization of fuzzy automata with membership values in complete residuated lattices. Information Sciences, 178:164-180, 2008.

[16] F. Katritzke. Refinements of Data Compression Using Weighted Finite Automata. PhD thesis, Siegen University, 2001.

[17] G.J. Klir and B. Yuan. Fuzzy Sets and Fuzzy Logic, Theory and Application. Prentice-Hall, Englewood Cliffs, NJ, 1995.

[18] K. Knight and J. May. Applications of weighted automata in natural language processing. In M. Droste, W. Kuich, and H. Vogler, editors, Handbook of Weighted Automata, chapter 14. Springer-Verlag, 2009. to appear.

[19] K.V. Krishna. Near-Semirings: Theory and Applications. PhD thesis, IIT Delhi, New Delhi, India, 2005.

[20] W. Kuich and A. Salomaa. Semirings, Automata, Languages, volume 5 of Monogr. Theoret. Comput. Sci. EATCS Ser. Springer-Verlag, 1986.

[21] T. Lengauer and D. Theune. Unstructured Path Problems and the Making of Semirings. In Algorithms and Data Structures, Proceedings WADS'91, Ottawa, Canada, number 519 in Lecture Notes in Comput. Sci., pages 189200, 1991.

[22] Y. Li. Approximation and robustness of fuzzy finite automata. International Journal of Approximate Reasoning, 47:247-257, 2008.

[23] Y. Li and W. Pedrycz. Fuzzy finite automata and fuzzy regular expressions with membership values in lattice-ordered monoids. Fuzzy Sets and Systems, 156:68-92, 2005.

[24] Y. Li and W. Pedrycz. Minimization of lattice finite automata and its application to the decomposition of lattice languages. Fuzzy Sets and Systems, 158:1423-1436, 2007.

[25] M. Mohri. Finite-state transducers in language and speech processing. Computational Linguistics, 23(2):269-311, 1997.

[26] M. Mohri. Minimization algorithms for sequential transducers. Theoretical Computer Science, 234:177-201, 2000.

[27] M. Mohri, F. Pereira, and M. Riley. The design principles of a weighted finite-state transducer library. Theoret. Comp. Science, 231:17-32, 2000.

[28] J. Sakarovitch. Éléments de Théorie des Automates. Vuibert, 2003.

[29] A. Salomaa and M. Soittola. Automata-Theoretic Aspects of Formal Power Series. Texts and Monographs in Computer Science, Springer-Verlag, 1978. 
[30] M.P. Schützenberger. On the definition of a family of automata. Inf. and Control, 4:245-270, 1961.

[31] B. Šešelja, A. Tepavčević, and H. Vogler. A note on cut-worthyness of recognizable tree series. Fuzzy Sets and Systems, 2008. accepted for publication.

[32] W.G. van Hoorn and B. van Oozelaa. Fundamental notions in the theory of seminearrings. Compositio Math., 18:65-78, 1967.

[33] W. Wechler. The Concept of Fuzziness in Automata and Language Theory. Studien zur Algebra und ihre Anwendungen. Akademie-Verlag Berlin, 5. edition, 1978. 